\title{
COMBINED ANDROGEN BLOCKADE RESPONSIVE PROSTATE CARCINOMA WITH EXTENSIVE NODAL METASTASES WITH VIRCHOW'S NODES
}

\author{
Senthilvel Arumugam1, Saravanan Kanakasabapathy'2, Matheen Jaffer Farman ${ }^{3}$ \\ ${ }^{1}$ Senior Assistant Professor, Department of Urology, Government Royapettah Hospital, Chennai. \\ ${ }^{2}$ HOD, Department of Urology, Government Royapettah Hospital, Chennai. \\ ${ }^{3}$ Resident, Department of Urology, Government Royapettah Hospital, Chennai.
}

ABSTRACT

\section{BACKGROUND}

Carcinoma prostate is one of the most common cancer in an elderly male. With the peak incidence between 70 and 74 years of age, less than $1 \%$ of patients with prostate cancer are younger than 50 years of age. Metastasis from prostate cancer occurs via local spread, lymphatic spread and haematogenously to the axial skeleton. Here, we present a case report of advanced prostate cancer presenting as cervical lymph node enlargement, which is a rare presentation.

\section{MATERIALS AND METHODS}

We report a case of prostate cancer presenting with left supraclavicular nodal enlargement without any urological symptom. On examination, hard nodular prostate was detected. Serum prostate specific antigen (PSA) and alkaline phosphatase were elevated. Imaging studies showed bulky lymph nodal enlargement in mediastinum and para-aortic region. Bone scintigraphy revealed extensive skeletal metastasis. Biopsy of supraclavicular node was positive for PSA and biopsy of prostate showed adenocarcinoma (Gleason 5+4). Patient responded well to complete androgen blockade.

\section{RESULTS}

Carcinoma of prostate usually metastasises to regional group of lymph nodes and distant metastases to supraclavicular and mediastinal nodes are rarely reported, especially as initial presentation.

\section{CONCLUSION}

Although metastasis to supraclavicular node is rare in prostate cancer, rectal examination and PSA should be done in elderly males with a high index of suspicion to make the diagnosis in this clinical setting.

\section{KEYWORDS}

Virchow's Node, Carcinoma, Prostate, Androgen, Blockade.

HOW TO CITE THIS ARTICLE: Arumugam S, Kanakasabapathy S, Farman MJ.Combined androgen blockade responsive prostate carcinoma with extensive nodal metastases with Virchow's' nodes. J. Evolution Med. Dent. Sci. 2017;6(8):661-664, DOI: $10.14260 / \mathrm{Jemds} / 2017 / 142$

\section{BACKGROUND}

A 70-year-old male admitted in our institution with neck swelling in left side for one month. He had no other symptoms other than malaise, loss of appetite and weight. Physical examination showed $7 \times 5 \mathrm{~cm}$ hard left supraclavicular and posterior triangle lymph nodes, immobile and nontender. Other general examination was normal. Examination of the abdomen was normal. No abnormalities detected in cardiovascular and respiratory examination. External genitalia was normal. Per rectal examination showed hard nodular prostate with normal tone. Complete haemogram and renal function tests were within normal limits. Serum Prostate specific antigen (PSA) was more than $400 \mathrm{ng} / \mathrm{mL}$ and serum alkaline phosphatase was increased.

Financial or Other, Competing Interest: None.

Submission 17-10-2016, Peer Review 10-11-2016,

Acceptance 16-11-2016, Published 25-01-2017.

Corresponding Author:

Dr. Senthilvel Arumugam,

Department of Urology,

Government Royapettah Hospital,

Westcott Road, Royapettah,

Chennai-600014.

E-mail: arusenthil2014@gmail.com

DOI: $10.14260 /$ jemds $/ 2017 / 142$

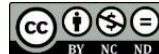

Plain chest radiography demonstrated mediastinal widening. Transrectal and Ultrasonogram of abdomen and pelvis showed hypoechoic lesion in prostate infiltrating the trigone in left side causing left hydroureteronephrosis. Computed tomography of the chest, abdomen and pelvis showed multiple lymph nodal enlargement in mediastinum, and bulky mass of paraaortic and paracaval lymph nodes with left hydroureteronephrosis. Fine needle aspiration of neck node showed adenomatous metastatic deposits. Biopsy from the supraclavicular node showed positive for prostate specific antigen. Transrectal biopsy of the prostate revealed poorly differentiated adenocarcinoma (Gleason score 5+4). Bone scintigraphy showed extensive skeletal metastasis. Prostate cancer was staged according to TNM classification as T4N1M1. Bilateral orchiectomy was done and started on hormonal therapy with antiandrogens (Tablet Bicalutamide $50 \mathrm{mg}$ daily). Patient responded well to the hormonal therapy with drastic decrease in the size of nodes and serum Prostate specific antigen levels to $53 \mathrm{ng} / \mathrm{mL}$ on $14^{\text {th }}$ post-operative day. 


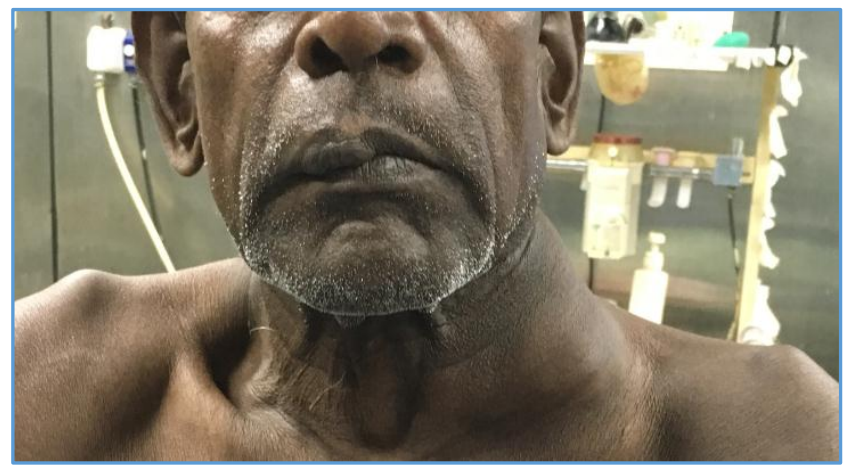

Figure 1. Virchow's Nodes in Carcinoma Prostate Patient

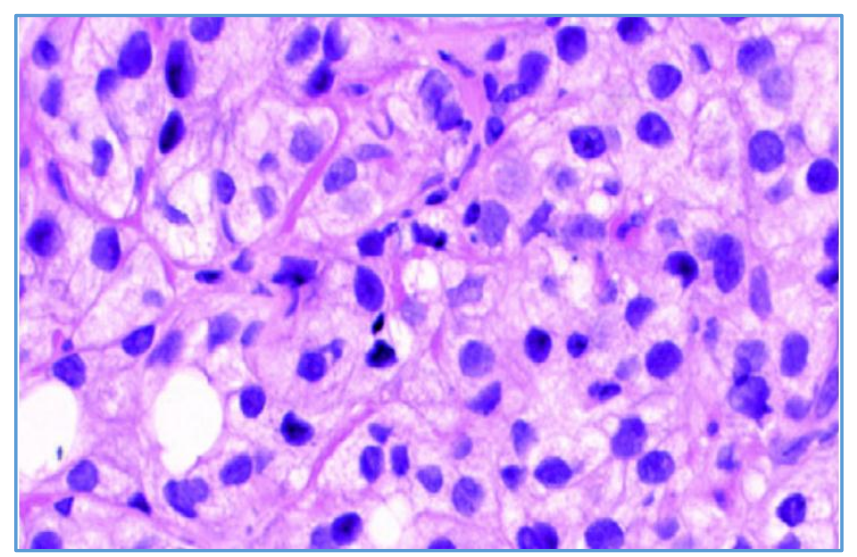

Figure 2. HPE - Adenocarcinoma of Prostate

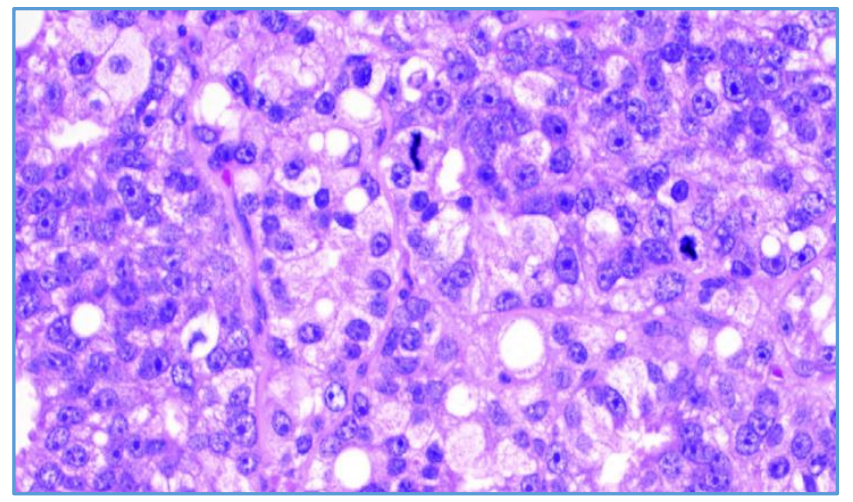

Figure 3. Biopsy of Virchow's Nodes - Positive for PSA

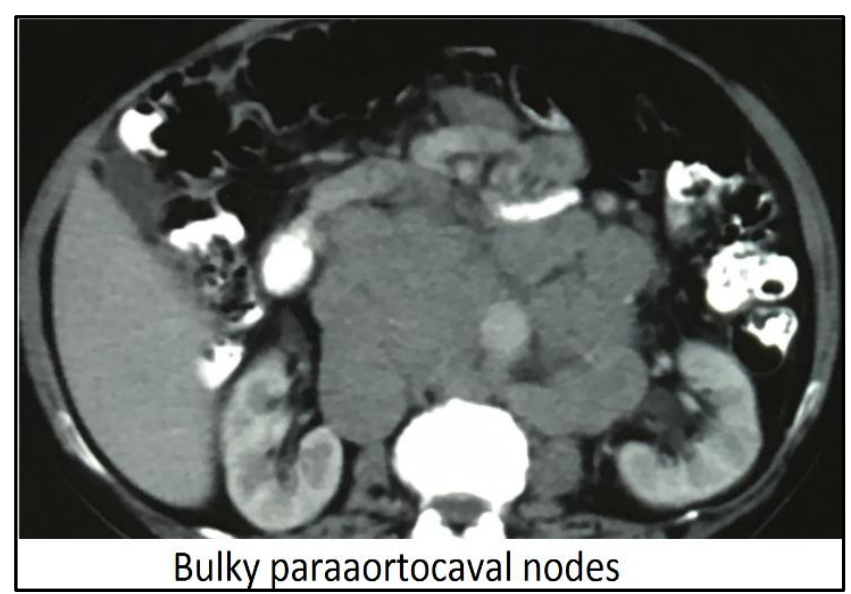

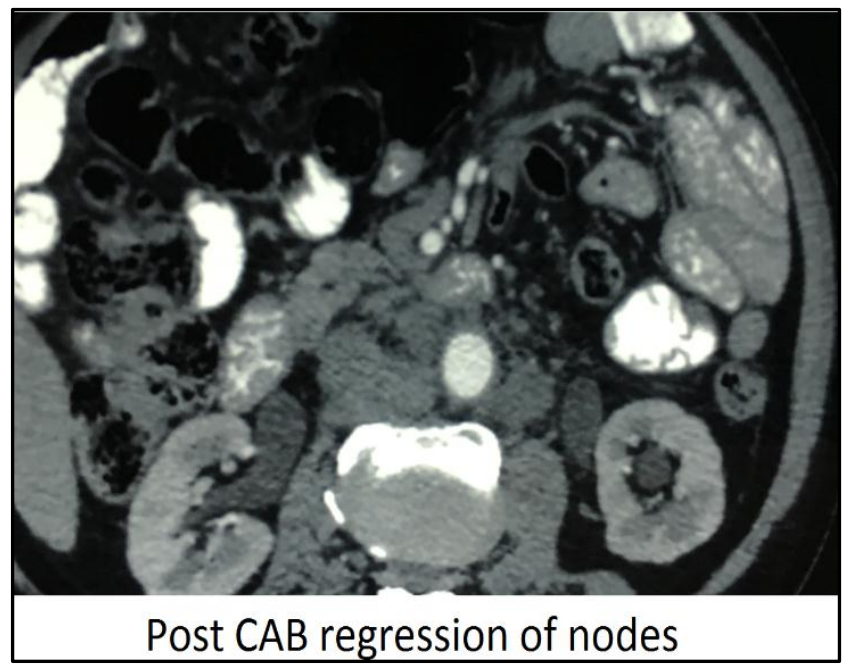
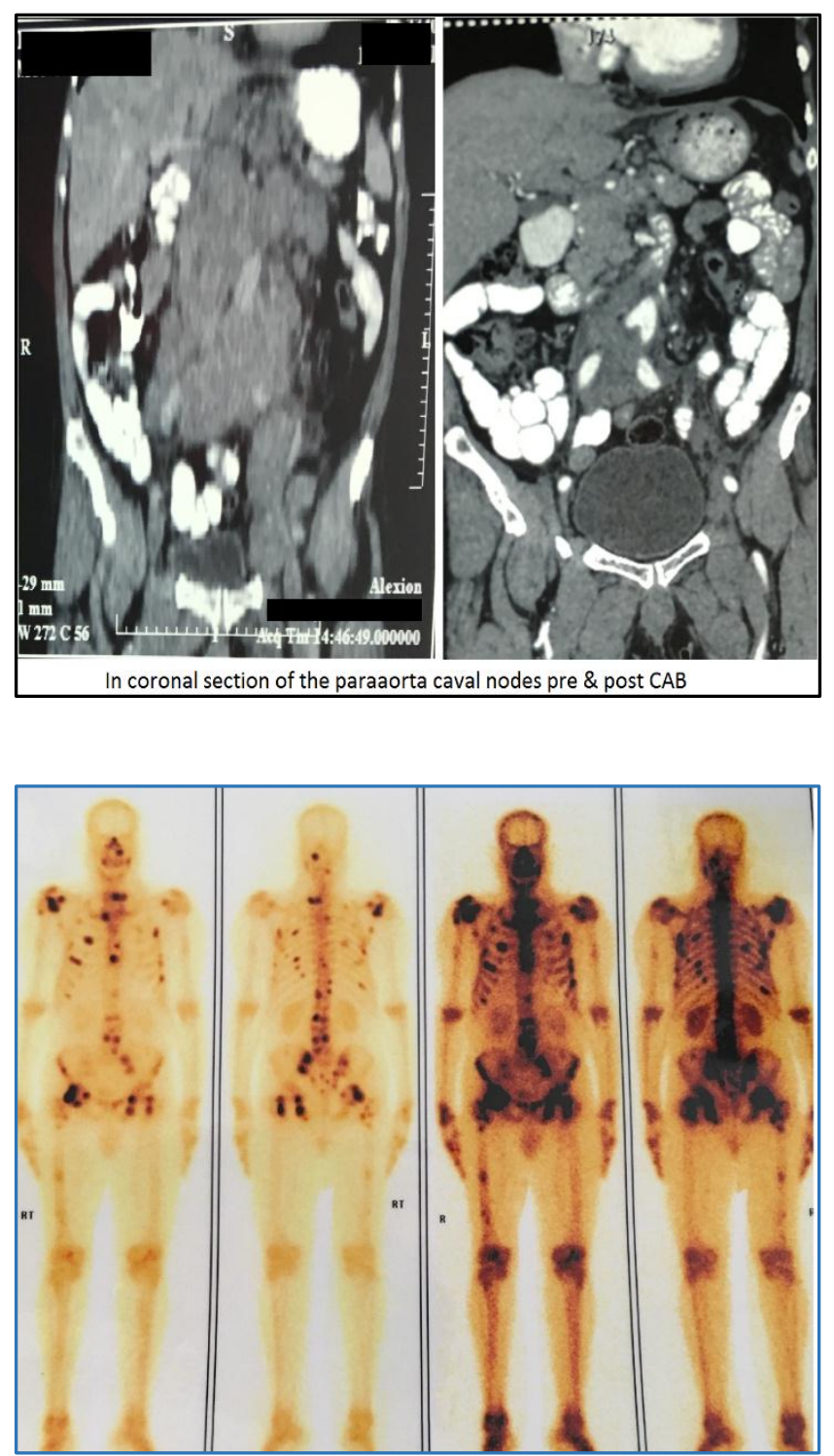

Figure 4. Bone Scan Showing Multiple Skeletal Metastasis 


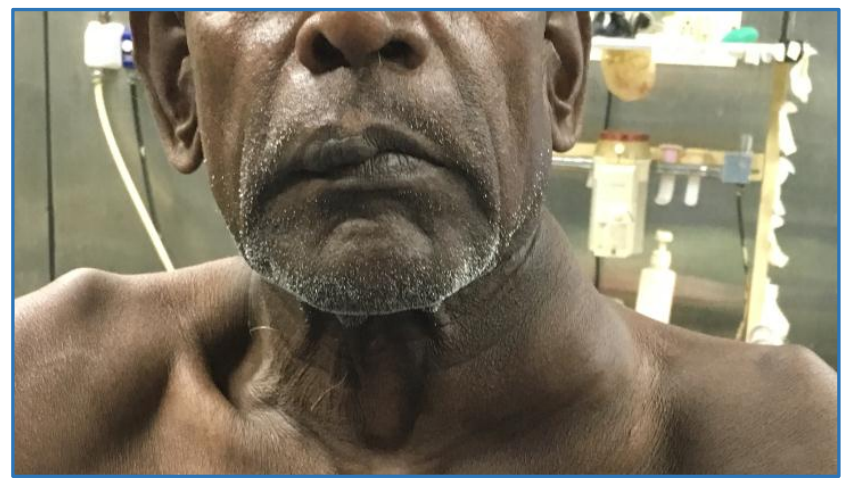

Figure 5. Before Orchiectomy

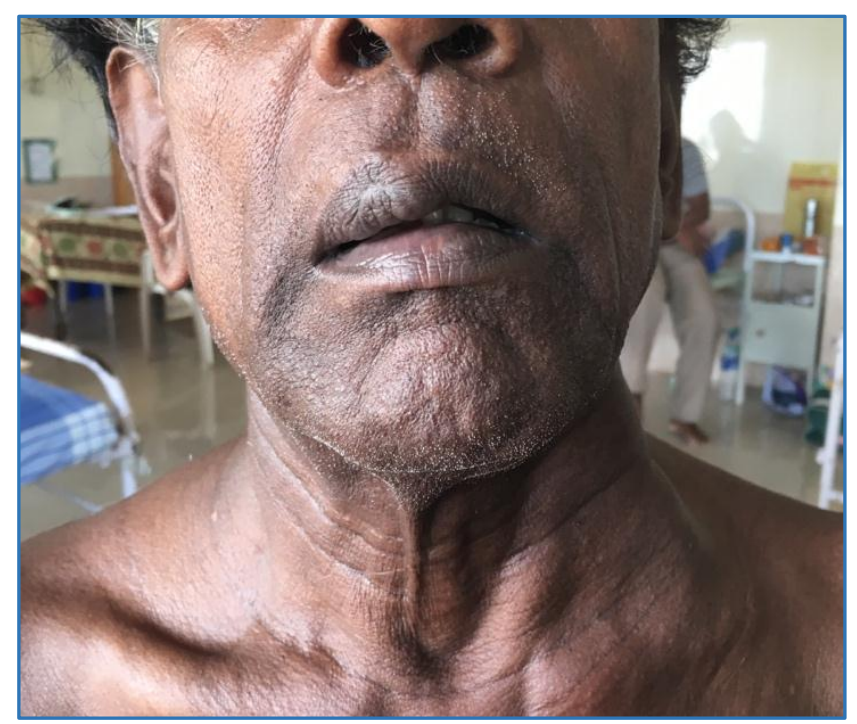

Figure 6. After Orchiectomy

\section{DISCUSSION}

Carcinoma prostate is one of the most common cancer in elderly males. With the peak incidence between the ages of 70 and 74 years, less than $1.0 \%$ of all patients with prostate cancer are younger than 50 years of age. ${ }^{1}$ Metastasis from prostate cancer most commonly occurs via lymphatic spread to the regional lymph nodes, direct invasion to the pelvic organs or systemically to the axial skeleton. ${ }^{2}$ The local growth of prostate may cause symptoms, such as obstruction and irritation, when voiding. Cervical nodal enlargement in the elderly is usually a reflection of manifestation of lymphoma or a metastatic dissemination from either squamous cell carcinoma or adenocarcinoma. Most cancers that present with cervical lymphadenopathy are derived from head and neck malignancies involving the mucosal surface of the aerodigestive tract, thyroid glands. The most common nonhead or neck primary cancers metastatic to cervical chain and supraclavicular nodes are lung, breast, kidney, upper gastrointestinal tract. The rich venous plexus of Batson is implicated as the route of haematogenous dissemination of prostatic adenocarcinoma to the pelvic bones, femur, lumbar spine, thoracic spine and ribs.

Superficial Lymph node metastasis is infrequent and a rare mode of initial presentation in carcinoma prostate. ${ }^{3}$ The incidence of metastasis to supraclavicular node is $0.3-0.5 \%$ in carcinoma prostate. Batson postulated that head and neck metastases from prostate cancer occur due to haematogenous spread via the vertebral venous system (Batson plexus).
However, the haematogenous dissemination fails to explain the predilection of this carcinoma to metastasise to the left cervical region, whilst right side involvement is extremely uncommon. Prostate is richly supplied by lymphatics. There is typical stepwise progression. This involves the regional lymph nodes which drain into obturator, hypogastric and presacral nodes and from these to the iliac, paraaortic, cisterna chyli and thoracic duct. Finally, the lymphatic drainage enters the systemic blood circulation via the left subclavian vein. Some authors have postulated that tumour cells can lodge in the left supraclavicular and cervical nodes by retrograde spread due to the proximity of these nodes with the point of entry of thoracic duct into the left subclavian vein.

In this case, left neck nodes involving posterior triangle, supraclavicular nodes, paraaortocaval lymph nodes were enlarged. Most cases were lymphomas, adenocarcinoma arising from gastrointestinal organs and ovaries. But the involvement of posterior triangle cervical nodes is very rare in prostate cancer. There is presence of extensive bony metastasis in this patient. Epstein found that $40 \%$ of patients had no evidence of bony metastases, $28 \%$ had normal serum acid phosphatase levels, and $45 \%$ had no prostatic abnormalities on digital rectal exam. Jones and Anthony found that PSA or prostate specific acid phosphatase levels were elevated in only five of nine patients with cervical metastases. The possibility that not all patients may have elevated biochemical markers for prostate cancer emphasises the importance of an appropriate tissue diagnosis. While the patient in this case report had an elevated PSA, the ultimate diagnosis was established by immunohistochemistry with PSA staining on tissue obtained by biopsy.

Butler et $\mathrm{al}^{4}$ described 19 patients with prostate carcinoma presenting initially with enlargement of supraclavicular lymph nodes. They noted that left-sided supraclavicular nodes were much more commonly involved than those on the right. The diagnosis was confirmed by prostate biopsy in 14 patients. They noted that only $42 \%$ had an abnormal digital rectal examination. Our patient had an abnormal DRE and a markedly elevated serum PSA.

Woo et al ${ }^{5}$ described a 76-year-old patient presenting with supraclavicular lymphadenopathy. They recorded a normal prostate on digital rectal examination. A PSA performed several days after admission was raised at $326 \mathrm{ng} / \mathrm{mL}$ and a fine needle biopsy of the neck mass confirmed a prostate adenocarcinoma.

Cho et $\mathrm{al}^{6}$ reported 26 cases of metastatic prostate carcinoma in supradiaphragmatic lymph nodes (15 of these were supraclavicular lymph nodes) in which only 7 cases had a history of prostate carcinoma; they noted that $58 \%$ had abnormal rectal examinations and $42 \%$ had normal rectal examination, $35 \%$ had no bony metastases, and $24 \%$ had normal serum acid phosphatase.

Saeter et al 7 reported that in 35 patients with non-regional lymphatic spread from prostate carcinoma, the left supraclavicular fossa was the most common site of spread in $69 \%$ of cases, $80 \%$ of the patients had elevated acid phosphatase, and $75 \%$ had abnormal DRE.

\section{CONCLUSION}

In this case, there is an involvement of cervical, mediastinal, bulky paraaortic nodes with extensive bony metastasis. Although metastases to the supraclavicular lymph nodes are 
rare in prostate cancer patients, rectal examination and PSA should be done in investigating elderly males with lymphadenopathy.

\section{REFERENCES}

[1] Coffey DS. Prostate cancer: an overview of an increasing dilemma. Cancer 1993;71(3 Suppl):880-6.

[2] Venable DD, Hastings D, Misra RP. Unusual metastatic patterns of prostate adenocarcinoma. J Urol 1983;130(5):980-5.

[3] Dick VS. Carcinoma of the prostate gland with metastases. Surg Clin North Am 1962;42:771-7.
[4] Butler JJ, Howe CD, Johnson DE. Enlargement of supraclavicular lymph nodes as the initial sign of prostate carcinoma. Cancer 1971;27(5):1055-63.

[5] Woo K, Weiczorek R, Torre P, et al. Prostate adenocarcinoma presenting as a large supraclavicular mass. Rev Urol 2001;3(2):102-5.

[6] Cho KR, Epstein JI. Metastatic prostate carcinoma to supradiaphragmatic lymph nodes. A clinicopathologic and immunohistochemical study. Am J Surg Pathol 1987;11(6):457-63.

[7] Saeter G, Fossa SD, Ous S, et al. Carcinoma of the prostate with soft tissue or non-regional lymphatic metastases at the time of diagnosis: a review of 47 cases. Br J Urol 1984;56(4):385-90. 